1. High velocity flow jet in an up-stream stenosis in the transverse sinus directed at the SSD opening,

2. Flow jet into the SSD along the long axis of the SSD, either anteriorly or laterally directed,

3. Vortex of flow in the SSD,

4. Prominent vortex component of flow in the sigmoid sinus downstream from SSD,

5. Vortex of flow in the jugular bulb

Three of the patients had simulated post-coil treatment models developed excluding the SSD from the models. CFD showed no flow in SSD and decreased vortex component of flow in the sigmoid sinus downstream from the SSD.

Conclusion PT caused by SSD may be caused by a unique flow pattern in the SSD and sinuses as visualized on both MRV and CFD.

Disclosures M. Amans: None. E. Kao: None. S. Kefayati: None. K. Meisel: None. F. Faraji: None. C. Glastonbury: None. M. Ballweber: None. V. Halbach: None. D. Saloner: None.

\section{0-033 JUGULAR VEIN FLOW PATTERNS IN PATIENTS WITH PULSATILE TINNITUS USING COMPUTATIONAL FLUID DYNAMICS}

${ }^{1} \mathrm{E}$ Kao, ${ }^{1} \mathrm{~S}$ Kefayati, ${ }^{2} \mathrm{~K}$ Meisel, ${ }^{1} \mathrm{M}$ Ballweber, ${ }^{1} \mathrm{~F}$ Faraji, ${ }^{1} \mathrm{~V}$ Halbach, ${ }^{1} \mathrm{D}$ Saloner, ${ }^{1} \mathrm{M}$ Amans. ${ }^{1}$ Radiology, UCSF, San Francisco, CA; ${ }^{2}$ Neurology, UCSF, San Francisco, CA

\subsection{6/neurintsurg-2016-012589.33}

Introduction/purpose Aberrations of venous anatomy can cause pulsatile tinnitus (PT). However, venous anatomy variation in patients without pulsatile tinnitus (PT) is extremely variable. Conventional imaging modalities, including cerebral angiography, provide little insight into the complex flow patterns in the cerebral veins. The aim of this study was to use subjectspecific contrast-enhanced MRA (CE-MRA) determined anatomy, and MRV-based inlet flow conditions to develop computational fluid dynamics (CFD) models of flow in subjects with suspected venous pulsatile tinnitus and subjects without pulsatile tinnitus to investigate how the geometry of the jugular vein affects flow.

Materials and methods 7 jugular veins (4 normal, 3 with pulsatile tinnitus) were imaged with CE-MRA. 2 D phase contrast MRV was also acquired transverse to the sigmoid sinus to determine inlet flow conditions. Surfaces were segmented using VMTK (Orobix, Bergamo, Italy) and Geomagic Design $\mathrm{X}$ (Geomagic, Rock Hill, USA). Tetrahedral meshing was also performed in VMTK, using a target edge-length of $0.6 \mathrm{~mm}$. CFD simulations were performed in FLUENT (ANSYS, Canonsburg, USA), using flow values obtained from the literature and in vivo measurements. Flow patterns in the jugular vein were characterized by their vortex core-lines, which were extracted from simulation data using Ensight (CEI, Apex, USA). Additional flow-parameters were calculated in MATLAB (Mathworks, Natick, USA) and pathlines visualized using Paraview (Kitware, New York, USA).

Results Patients with suspected venous PT had flow distinct patterns from those in normal subjects. Non-PT flow was characterized by organized redirection of flow from the sigmoid sinus along the curvature of the bulb into the jugular vein (A) with vortex cores in the jugular bulb (C). PT flow was characterized by larger helical flow structures throughout the proximal jugular vein created by flow directed perpendicular to the sigmoid sinus flow (B) and vortex cores that were more diffusely organized (D). High flow rates, and even turbulence, were noted in the proximal jugular vein near the level of the carotid bifurcation - where there was often pronounced narrowing of the jugular.

Conclusion The geometry of the jugular vein significantly affects the position, size, and length of the vortex cores. Our results suggest a link between geometry, flow, and PT.

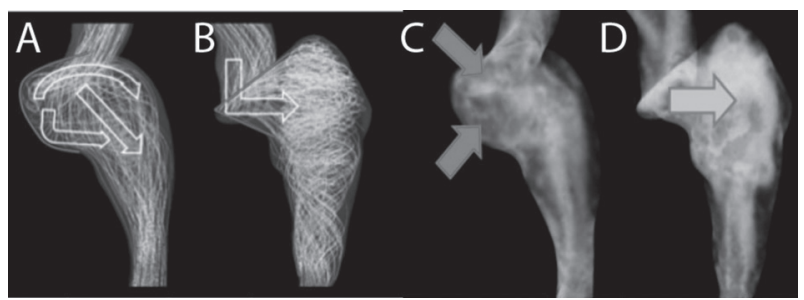

Abstract 0-033 Figure 1 Visualization of streamlines (A, B) and vortex cores by swirling strength $(C, D)$ in internal jugular veins of subjects without $(A, C)$ and with $(B, D)$ PT. White arrows $(A, B)$ reperent the general re-direction of flow from the sigmoid sinus into the jagular vein as dictated by the shape of the junction with the jugular bulb (red lines). Subjects without PT have strong vortex cores in jugular bulb (C, red arrows), while those with PT have larger, more diffuse vortex cores (D) that encompass nearly the entire proximal jugular vein (blue arrow).

Disclosures E. Kao: None. S. Kefayati: None. K. Meisel: None. M. Ballweber: None. F. Faraji: None. V. Halbach: None. D. Saloner: None. M. Amans: None.

\section{0-034 ABCIXIMAB THERAPY FOR THROMBOEMBOLIC COMPLICATIONS OF NEUROENDOVASCULAR PROCEDURES}

${ }^{1} \mathrm{~A}$ Kansagra, ${ }^{1} \mathrm{~T}$ Madaelil, ${ }^{1} \mathrm{D}$ Cross, III, ${ }^{1} \mathrm{C}$ Moran, ${ }^{2} \mathrm{C}$ Derdeyn. ${ }^{1}$ Washington University School of Medicine, Saint Louis, MO; ${ }^{2}$ University of lowa Hospitals and Clinics, Iowa City, IA

\subsection{6/neurintsurg-2016-012589.34}

Background Thromboembolic complications sustained during neuroendovascular procedures can result in postoperative infarcts if not promptly recognized and treated. Treatment commonly involves glycoprotein IIb/IIIa inhibitors such as abciximab. We aimed to retrospectively review angiographic and clinical outcomes following abciximab administration for thromboembolic complications at our institution.

Methods Neuroendovascular cases with thromboembolic complications treated with abciximab over a 132 month period were identified using a search of a comprehensive, prospectively maintained case $\log$ and all angiography records for the terms "abciximab" or "ReoPro." Intraoperative intra-arterial (IA) administration typically involved slow infusion of $10 \mathrm{mg}$ abciximab over 5 to 10 minutes. Intraoperative intravenous (IV) administration $0.25 \mathrm{mg} / \mathrm{kg}$ abciximab. Postoperative IV infusion typically involved $10 \mathrm{mcg} / \mathrm{min}$ infusion of abciximab. All relevant clinical notes and neuroimaging were reviewed.

Results Of 19,566 neuroendovascular procedures performed during the review period, $48(0.25 \%)$ involved abciximab administration for thromboembolic complications. 65\% (31/48) involved IA administration, 21\% (10/48) were IV only, and $15 \%$ (7/48) were combined IA and IV. Intraoperative treatment was supplemented with postoperative abciximab infusion in $13 \%(6 / 48)$ patients. Angiographic improvement was seen in $92 \%(44 / 48)$ cases, including 65\% (31/48) with complete 


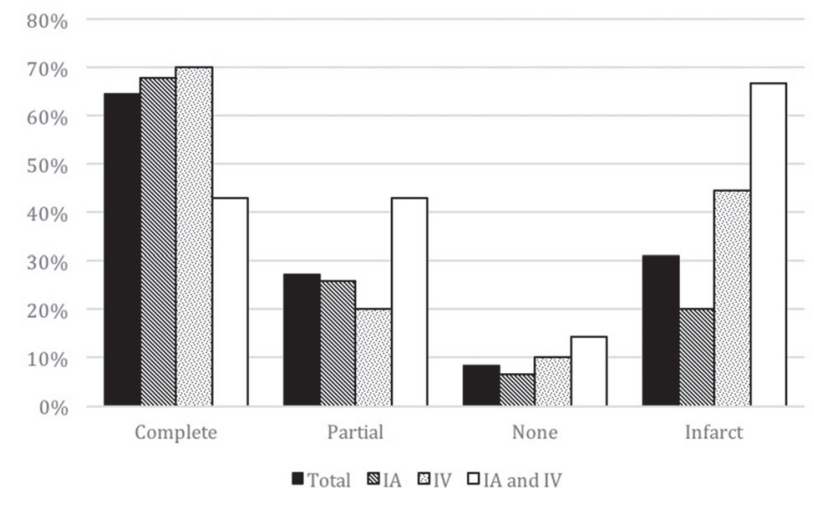

Abstract 0-034 Figure 1 Frequency of complete, partial, or no angiographic improvement and frequency of postoperative infarction following intraoperative abciximab administration

angiographic resolution (Figure 1). Only 8\% (4/48) cases failed to improve angiographically. Angiographic outcomes were similar in patients who received IA, IV, or combined IA and IV administration of abciximab intraoperatively $(p=0.58)$, or patients who did or did not receive continuous IV infusion of abciximab postoperatively $(p=0.11)$. Postoperative infarction was seen in 20\% (5/25), 44\% (4/9), and 67\% (4/6) following IA, IV, and combined IA and IV abxicimab, respectively $(p=0.06)$ (Figure 1$)$. The rate of postoperative infarction was $33 \%(2 / 6)$ in patients who received continuous IV infusion of abciximab postoperatively and 32\% (11/34) in those who did not $(p=1.00)$. Postoperative infarction developed in $8 \%(2 / 24)$ patients with complete angiographic improvement, compared to $69 \%(11 / 16)$ in patients with partial or no improvement $(p<0.0001)$.

Conclusion Abciximab administration is an effective method of treating thromboembolic complications of neuroendovascular procedures. Angiographic outcomes were not appreciably different between different routes of abciximab administration. Infarction was least common in patients treated with IA abciximab; the addition of postoperative abciximab infusion did not affect the rate of infarction. No or partial angiographic improvement was associated with significantly higher rates of postoperative infarction than complete angiographic improvement.

Disclosures A. Kansagra: None. T. Madaelil: None. D. Cross: None. C. Moran: 2; C; Medtronic Neurovascular. 3; C; Medtronic Neurovascular. C. Derdeyn: None.

\section{0-035 SAFE (SAFETY AND EFFICACY ANALYSIS OF FRED EMBOLIC DEVICE IN ANEURYSM TREATMENT): STUDY DESIGN AND PRELIMINARY RESULTS}

L Pierot, J Gauvrit, J Lejeune, A Derelle, J Gabrillargues. Radiology, Hôpital MaisonBlanche, Reims, France

\subsection{6/neurintsurg-2016-012589.35}

Purpose Flow diversion is now a well-established endovascular technique for the treatment of intracranial aneurysms. FRED and FRED $\mathrm{Jr}$ are dual-layer, self-expanding nickel titanium flow-diverters. SAFE is a prospective, multicenter study conducted in 14 French and Belgian centers to evaluate the safety and efficacy of these devices.

Materials and methods Only aneurysms located in the anterior circulation were included. The primary efficacy endpoint is the rate of complete aneurysm occlusion at 6 months without associated stenosis of the parent vessel. The primary safety endpoint is the rate of morbidity $(\mathrm{mRS}>2)$ and mortality at 6 months. Adverse events as well as anatomical results will be independently evaluated. According to the endpoints, the target population is 85 patients, now extended to $95 / 100$.

Results Inclusions started in July 2014. End of February 2016, 92 patients with 92 aneurysms were included. The expectation is to have inclusions completed end of April 2016. Most patients were females $(80 / 92,87.0 \%)$. All but one patient were mRS 0 or 1 before the treatment.

Among the 92 aneurysms, 22 (23.9\%) were aneurysm remnant after a previous treatment and the 70 others $(76.1 \%)$ were unruptured. Aneurysm locations were internal carotid artery $(\mathrm{n}=83$, $90.2 \%)$, anterior communicating artery $(\mathrm{n}=6,6.5 \%)$, and middle cerebral artery $(\mathrm{n}=3,3.3 \%)$. Aneurysm size was $<10 \mathrm{~mm}$ in 57 aneurysms (62.0\%), 10 to $24 \mathrm{~mm}$ in 31 aneurysms (33.7\%), and $>24 \mathrm{~mm}$ in 3 aneurysms (3.3\%).

Neck size was $<4 \mathrm{~mm}$ in 24/91 aneurysms (26.4\%) and $>$ or $=4 \mathrm{~mm}$ in $67 / 91$ aneurysms $(73.6 \%)$.

Placement of the flow diverter was achieved in all cases. Intra-operative events were reported in $7 / 92$ patients $(7.6 \%)$, including technical problems $(\mathrm{n}=3)$, thromboembolic events $(\mathrm{n}=3)$, and others $(\mathrm{n}=1)$. Postoperative events, morbidity, and mortality are also analyzed. Preliminary anatomical results (at 6 months) are presented.

Conclusion SAFE is an ongoing GCP (Good Clinical Practice) study aiming to evaluate safety and efficacy of FRED flow diverter in aneurysm treatment. Inclusions are close from completion. As of now, the safety of the FRED as evaluated by the CEC seems to be in line with published results for Flow Diversion technique.

Disclosures L. Pierot: 2; C; Microvention. J. Gauvrit: 2; C; Microvention. J. Lejeune: 2; C; Microvention. A. Derelle: 2; C; Microvention. J. Gabrillargues: 2; C; Microvention.

\section{0-036 RUPTURED ANEURYSMS OF COLLATERAL VESSELS IN ADULT-ONSET MOYAMOYA DISEASE WITH HEMORRHAGIC PRESENTATION}

${ }^{1} Y$ Cho, ${ }^{2} \mathrm{H}$ Kang, ${ }^{1} \mathrm{M}$ Han. ${ }^{1}$ Radiology, Seoul National University Hospital, Seoul, Republic of Korea; ${ }^{2}$ Neurosurgery, Seoul National University Hospital, Seoul, Republic of Korea

\subsection{6/neurintsurg-2016-012589.36}

Purpose Strategies for treating intracranial aneurysms of collateral vessels in adult-onset moyamoya disease (MMD) remain unclear, because overall case numbers are limited and data on long-term outcomes are lacking. The aim of this study was to assess clinical and anatomic outcomes of such aneurysms in adult MMD sufferers who present with hemorrhage.

Methods Of the 77 adult patients consecutively enrolled between January, 2003 and December, 2014 in the MMD registry at a single institution, those presenting with hemorrhage and followed for $>12$ months were studied. Aneurysms involving collateral vessels at sites of hemorrhage were considered culprit lesions.

Results Aneurysms of collateral vessels in 19 patients (19/77, $24.7 \%$ ) were confirmed as ruptured by conventional angiography. In five of these patients, obliterative endovascular embolization was successfully performed. The other 14 patients were managed conservatively due to lesion inaccessibility. Follow-up imaging studies (13 available) confirmed later disappearance of 